\title{
Y Chromosomal SNP Analysis Using the Minisequencing Strategy in a Moroccan Population Samples
}

\author{
Rachid Aboukhalid ${ }^{1,4 *}$, Mehdi Bouabdellah ${ }^{1,4}$, Ronald Lai $^{2}$, Nicholas Bradford ${ }^{2}$, Hicham EL Ossmani ${ }^{3}$, Jalal Talbi ${ }^{3}$, Kaoutar Bentayebi $^{1}$,
} Driss Squalli ${ }^{4}$, Daniele Podini ${ }^{2}$ and Saaid Amzazi ${ }^{1}$

${ }^{1}$ Forensic Genetic Unit, Biochemistry and Immunology Laboratory, Mohammed V University, Faculty of Science, BP 1014, Avenue Ibn Batouta Agdal, Rabat, Morocco ${ }^{2}$ Department of Forensic Science, The George Washington University, 2100 Foxhall Road NW Washington, DC 20007, United States

${ }^{3}$ Laboratory of Anthropogenetics and Physiopathology, Department of Biology, University of Chouaïb Doukkali, Faculty of Science, El Jadida, Morocco

${ }^{4}$ GENOME Biotechnologies, Parc d'activité Oukacha, Imm E N¹6, Ain Sebaâ, Casablanca, Morocco

\begin{abstract}
The $\mathrm{Y}$ chromosome contains the largest non-recombining portion in the human genome. Y-Binary polymorphisms, also known as a single nucleotide polymorphisms (SNPs), are a series of biallelic polymorphisms occurring on the non-recombining region of the $\mathrm{Y}$ chromosome (NRY), which represent a precious tool for human evolutionary studies and, potentially, for forensic applications. Low mutation rate, paternal inheritance, and absence of recombination make Y-SNPs particularly suitable for the identification of stable paternal lineages and the reconstruction of an ancestral state from which to explore the evolution of humans. Also, these markers would allow inference of the paternal ancestry of unknown samples which could be useful in forensic applications. In the present study we analyzed 22 biallelic Y-SNPs in 159 males belonging to three ethnic groups (Arab $n=42$, Berber $n=67$ and Sahrawi $n=50$ ) from Morocco. A total of 10 different haplogroups were identified in this sample representative of Moroccan population. The most common $Y$ chromosome haplogroups is $\mathrm{E} 1 \mathrm{~b} 1 \mathrm{~b} 1, \mathrm{E} 1 \mathrm{~b} 1 \mathrm{~b} 1 \mathrm{~b}$ and $\mathrm{J} 1$ with frequencies of $56 \%, 49 \%$ and $10 \%$ respectively.
\end{abstract}

Keywords: Y-chromosomal SNP; Mini-sequencing; SNaPshot; Morocco

\section{Introduction}

Biallelic markers, such as single nucleotide polymorphisms (SNPs) and insertion/deletions (indels), represent an important class of markers on the Y-chromosome [1]. Y-chromosome SNPs (Y-SNPs) are mostly used in molecular anthropology for evolutionary research. Moreover, typing a sample's Y chromosome hapologroup allows paternal ancestry inference. This may be useful, in forensic applications, when a conventional Short Tandem Repeat (STR) profile, generated from DNA collected at a crime scene, does not match any of the identified suspects and doesn't "hit" any profile on the available databases.

The kingdom of Morocco is a country located in the northwestern corner of the African continent with coasts on the Atlantic Ocean and the Mediterranean Sea; it is bordered by Algeria to the east and Mauritania to the south. Modern-day Morocco is inhabited by three major ethnic groups (Arab, Berber and Sahrawi). Several dialects such as Arabic (Moroccan dialect or Darija), Berber (Tarifit, Tachelhit and Tamazight) and Sahrawi (El Hassania) are spoken in the country. The aim of the present study was to develop an assay to genotype a selected panel of Y-SNPs using single base extension assay (SBE), or minisequencing [2], in order to determine the most frequent Y-chromosomal haplogroup in Moroccan population. The analysis of single nucleotide polymorphisms (SNPs) is a promising application in forensic casework; since the forensic scientists is often faced with degraded and/or very low amounts of DNA.

\section{Materials and Methods}

\section{Population}

Buccal swabs were collected from 159 unrelated healthy adult men belonging to the three ethnic groups (Arab $n=42$, Berber $n$ $=67$ and Sahrawi $n=50$ ). Informed consent was obtained from all participants in this study, and information about the geographical origin of their grand-parents and about their first language was recorded.

\section{DNA Isolation}

Genomic DNA was extracted from buccal swab punches, using DNA IQ ${ }^{\mathrm{TM}}$ System (Promega; Madison, Wisconsin) on the Biomek ${ }^{\circledR}$ 2000 (Beckman Coulter, Brea, CA) robotic platform according to manufacturer's instructions.

\section{Y-SNP selection and multiplex design}

Assay design and development together with sample testing were conducted in the Forensic Molecular Biology Laboratory of the Forensic Sciences Department of The George Washington University. A total of $22 \mathrm{Y}$ chromosome biallelic markers were selected for this study following a hierarchical strategy based on the phylogenetic tree of Y chromosome recognized by the Y Chromosome Consortium (YCC) [3]. Loci Nomenclature is based on Karafet et al. [4]. The reference sequence for each Y-SNP was taken from the International Society of Genetic Genealogy, Y-DNA Haplogroup Tree 2010 (http://www. isogg.org/tree). The 22 markers were genotyped in two heptaplex, one hexaplex, and one duplex PCR reactions. The Multiplex MY01 allows the detection of major clades (A-R), A-M91 (A), B-M60 (B), C-RPS4Y $_{711}$ (C), D-M174 (D), J-M267 (J1), I-M170 (I) and R-M207 (R). The Multiplexes MY02 E-P147 (E1), E-M132 (E1a), E-P189 (E1b1a), E-M215 (E1b1b), E-M81 (E1b1b1b), E-M54 (E2b), J-M172 (J2), MY03 J-L24 (J2a4h), J-M221 (J2b), R-L63 (R1a), R-M343 (R1b), R-M269 (R1b1b2),

*Corresponding author: Rachid Aboukhalid, Genetic Unit, Biochemistry and Immunology Laboratory, Mohammed V University, Faculty of Science, BP 1014 Avenue Ibn Batouta Agdal, Rabat, Morocco, GENOME Biotechnologies, Parc d'activité Oukacha, Imm E N 16, Ain Sebaâ, Casablanca, Morocco Tel: +212 6124 32 20; E-mail: a.rachid22@gmail.com

Received December 16, 2010; Accepted December 29, 2010; Published December 30, 2010

Citation: Aboukhalid R, Bouabdellah M, Lai R, Bradford N, El Ossmani H, et al. (2010) Y Chromosomal SNP Analysis Using the Minisequencing Strategy in a Moroccan Population Samples. J Forensic Res 1:113. doi:10.4172/2157-7145.1000113

Copyright: (c) 2010 Aboukhalid R, et al. This is an open-access article distributed under the terms of the Creative Commons Attribution License, which permits unrestricted use, distribution, and reproduction in any medium, provided the original author and source are credited. 
Citation: Aboukhalid R, Bouabdellah M, Lai R, Bradford N, El Ossmani H, et al. (2010) Y Chromosomal SNP Analysis Using the Minisequencing Strategy in a Moroccan Population Samples. J Forensic Res 1:113. doi:10.4172/2157-7145.1000113

T-M70 (T) and MY04 E-M35 (E1b1b1), E-M78 (E1b1b1a) subdivides haplogroups $\mathrm{E}, \mathrm{J}, \mathrm{R}$ and $\mathrm{T}$ to define the most frequent haplogroups in NorthWest Africa. Multiplex minisequencing primers were designed for each multiplex PCR. PCR primers used in the primer extension assays are listed in Table 1 . The extension primers designed in four multiplex sets labeled msMY01, msMY02, msMY03 and msMY04 are listed in Table 2.

\section{PCR amplification}

Amplifications were performed in a final volume of $25 \mu \mathrm{L}$ using a master mix containing 1X GeneAmp ${ }^{\circledR}$ PCR Gold buffer (Applied Biosystems, Foster City, CA), $400 \mu \mathrm{M}$ of dNTPs, $3 \mathrm{mM}$ of $\mathrm{MgCl}_{2}, 0.65$ $\mathrm{mg} / \mu \mathrm{L}$ bovine serum albumin (BSA), and 1.5 units of AmpliTaq Gold ${ }^{\circledR}$ DNA polymerase (Applied Biosystems). The thermal cycling program was carried out on a GenAmp 9700 (Applied Biosystems) using the following conditions: $95^{\circ} \mathrm{C}$ for 10 minutes, 35 cycles of $94^{\circ} \mathrm{C}$ for 30 seconds, $55^{\circ} \mathrm{C}$ for 30 seconds and $72^{\circ} \mathrm{C}$ for 30 seconds, and a final extension at $72^{\circ} \mathrm{C}$ for 10 minutes.

Following PCR amplification, unincorporated primers and dNTPs were removed by adding 1 unit of Exonuclease I (USB Corporation) and 1 unit of Shrimp Alkaline Phosphatase (SAP) to $5 \mu \mathrm{L}$ of PCR products and incubating a $37^{\circ} \mathrm{C}$ for 70 minutes followed by 20 minutes at $65^{\circ} \mathrm{C}$.

\section{Primer extension assay using fluorescence detection}

Multiplex primer extension reactions were carried out in a total volume of $15 \mu \mathrm{L}$ using $1.5 \mu \mathrm{L}$ of $\mathrm{ABI}$ Prism ${ }^{\circledR}$ SNaPshot ${ }^{\mathrm{TM}}$ multiplex kit mix (Applied Biosystems), $2 \mu \mathrm{L}$ of purified PCR product and 1.5 pmols of each extension primer. Extension reaction was as follows: 25 cycles of $96^{\circ} \mathrm{C}$ for 10 seconds, $50^{\circ} \mathrm{C}$ for 5 seconds, and $60^{\circ} \mathrm{C}$ for 30 seconds.

A $1.5 \mu \mathrm{L}$ of non-purified primer extension product was diluted in $9.8 \mu \mathrm{L} \mathrm{Hi}-\mathrm{Di}^{\mathrm{TM}}$ formamide and $0.2 \mu \mathrm{L}$ of GS120-LIZ internal lane standard (Applied Biosystems) and analyzed on ABI Prism ${ }^{\circledR} 3130$ Genetic Analyzer (Applied Biosystems). Separation was performed on a $36 \mathrm{~cm}$ array using POP $^{\mathrm{TM}}-7$ (Applied Biosystems). Results were analyzed using GeneMapper v 4.0 (Applied Biosystems).

\section{Results and Discussion}

A total of 10 haplogroups were observed across the 159 male samples that were tested (Table 3). The most common Y chromosome haplogroups in the combined Moroccan population resulted E1b1b1, E1b1b1b and $\mathrm{J} 1$ (56\%, 49\% and 10\% respectively).

The E-M215 derivative E1b1b1 is observed in significant frequencies in Moroccan samples. It's defined by the E-M35 SNP which appears to have originated in East Africa then migrating to the Near East and then on to North Africa and Europe.

Previous studies have shown that E1b1b1b (E-M81), formerly $\mathrm{E} 3 \mathrm{~b} 1 \mathrm{~b}$ or E3b2, is the predominant haplogroup in northwestern Africa [5 - 8]. Bosh et al. [9] obtained a higher frequency (64\%) of E-M81 (E1b1b1b) in Northwest African populations than the present study. This disparity could be explained by the difference of geographic area and circumstances of sample collection. However, according to our results and those of Bosh et al. [9], it seems that the E1b1b1b haplogroup is more characteristic of Sahrawi than Berbers. It is also seen, although at low frequency, in the Iberian Peninsula (4\%) and Sicily (3\%) due to recent gene flow from Northwest Africa through Gibraltar strait $[9-10]$. Nevertheless, this haplogroup (E1b1b1b) is not found in sub-Saharan Africa and its frequency sharply declines through the continent towards the east.

The J1 haplogroup, defined by the single nucleotide polymorphism (SNP) M267, is most frequent in the Arabian Peninsula especially in Yemen (76\%) [11].This could be attributed to the early medieval period during which the Semitic expansion spread J1 out of Arabia into North Africa [12].

The multiplex assays described herein were designed to explore the shallowest branches of Y chromosome haplogroups in Moroccan population. They could also be applied to human evolution and human genetists studies as well as to forensic casework for ancestry inferences.

\section{Acknowledgement}

We would like to express our sincere gratitude to the Moroccan-American Commission for Educational and Cultural Exchange (MACECE) and the Fulbrigh Program for the Fulbright scholarship, for supporting a part of our research in the United States. We would like, also, to thank the George Washington University and the Armed Forces DNA Identification Laboratory (AFDIL). We are greatly indebted to Kimberly Andreaggi Sturk, Jodi A. Irwin, Joni Johnson and Katherine Butler for discussion and technical assistance. Finally, an honorable mention goes to the support offered by GENOME Biotechnologies.

\section{Reference}

1. Butler JM (2003) Recent developments in Y-single tandem repeat and Y-single nucleotide polymorphism analysis. Forensic Sci Rev 15: 91.

2. Podini D, Vallone PM (2009) SNP genotyping using multiplex single base primer extension assays. Methods Mol Biol 578: 379-391.

3. $Y$ chromosome consortium (2002) A nomenclature system for the three of human Y-chromosomal binary haplogroups. 339-348.

4. Karafet TM, Mendez FL, Meilerman MB, Underhill PA, Zegura SL, et al. (2008) New binary polymorphisms reshape and increase resolution of the human $Y$ chromosomal haplogroup tree. Genome Res 18: 830-838.

5. Onofri V, Alessandrini F, Turchi C, Pesaresi M, Tagliabracci A (2008) Y-chromosome markers distribution in Northern Africa: High-resolution SNP and STR analysis in Tunisia and Morocco populations. Forensic Sci Int: Genetics 235-236.

6. Robino C, Crobu F, Gaetano D, Bekada A, Benhamamouch S, et al. (2008) Analysis of Y-Chromosomal SNP haplogroups and STR haplotypes in Algerian population sample. Int J Legal Med 122: 251-255.

7. Arredi B, Poloni ES, Paracchini S, Zerjal T, Fathallah DM, et al. (2004) A predominantly neolithic origin for Y-chromosomal DNA variation in North Africa. Am J Hum Genet 75: 338-345.

8. Cruciani F, La Fratta R, Santolamazza P, Sellitto D, Pascone R, et al. (2004) Phylogeographic Analysis of Haplogroup E3b (E-M215) Y Chromosomes Reveals Multiple Migratory Events Within and Out Of Africa. Am J Hum Genet 74: 1014-1022.

9. Bosch E, Calafell F Comas D, Oefner PJ, Underhill PA et al (2001) HighResolution Analysis of Human Y-Chromosome Variation Shows a Sharp Discontinuity and Limited Gene Flow between Northwestern Africa and the Iberian Peninsula. Am J Hum Genet 68: 1019-1029.

10. Adams SM, Bosch E, Balaresque PL, Ballereau SJ, Lee AC et al. (2008) The Genetic Legacy of Religious Diversity and Intolerance: Paternal Lineages of Christians, Jews, and Muslims in the Iberian Peninsula. Am J Hum Genet 83 725-736.

11. Alshamali F, Pereira L, Budowle B, Poloni ES, Currat M (2009) Local population structure in Arabian Peninsula revealed by Y-STR diversity. Hum Hered 68: 45-54.

12. Semino O, Magri C, Benuzzi G, Lin AA, Al-Zahery N, et al. (2004) Origin, diffusion, and differentiation of Y-chromosome haplogroups $\mathrm{E}$ and $\mathrm{J}$ : inferences on the neolithization of Europe and later migratory events in the Mediterranean area. Am J Hum Genet 74: 1023-1034. 